\title{
Processo de trabalho de supervisão de enfermagem em policlínicas: limites e possibilidades
}

\author{
Nursing supervision work process in \\ polyclinics: limits and possibilities
}

\section{Lidiana Almeida Sacramento ${ }^{1}$ (1) Wilton Nascimento Figueredo ${ }^{2}$ (b) Maria Lúcia Silva Servo ${ }^{3}$ (1) Tássia Teles Santana de Macêdo ${ }^{4}$ (1) Elionara Teixeira Boa Sorte Fernandes 5 (1)}

'Enfermeira (Feira de Santana). Bahia, Brasil. lidianadlalmeida@ymail.com ${ }^{2}$ Autor para correspondência. Universidade Estadual de Feira de Santana (Feira de Santana). Bahia, Brasil.wnfigueredo@uefs.br ${ }^{3}$ Universidade Estadual de Feira de Santana (Feira de Santana). Bahia, Brasil. mlsservo@uefs.br ${ }^{4}$ Escola Bahiana de Medicina e Saúde Pública (Salvador). Bahia, Brasil. tassiamacedo@bahiana.edu.br 5Universidade do Estado da Bahia (Guanambi). Bahia, Brasil. eboasorte@uneb.br

RESUMO | OBJETIVO: descrever os limites e as possibilidades do processo de trabalho de supervisão de enfermagem em policlínicas. MÉTODO: estudo descritivo, exploratório e de abordagem qualitativa, realizado em cinco policlínicas de uma grande cidade do Estado da Bahia, Brasil. As entrevistas semiestruturadas foram realizadas com as cinco supervisoras de enfermagem e analisadas pela de técnica análise de conteúdo. RESULTADOS: as supervisoras de enfermagem relataram como principais limites para o seu trabalho a resistência da equipe em participar de eventos educativos, relação precária entre demandas de usuários, insuficiência no quantitativo da equipe de enfermagem, dificuldade na regulação de usuários, recursos humanos e financeiros ineficazes. Entre as possibilidades foram relatadas a comunicação, o relacionamento interpessoal e a boa articulação entre a gestão e coordenação. CONCLUSÃO: os limites encontrados ratificam as dificuldades encontradas na gestão dos serviços de saúde, assim como atestam a complexidade e necessidade da supervisão de enfermagem em promover boas práticas para um cuidado qualificado.

DESCRITORES: Enfermagem. Supervisão de enfermagem. Centros de saúde. Administração de serviços de saúde. Organização e administração.
ABSTRACT | OBJECTIVE: to describe the limits and possibilities of the nursing supervision work process in polyclinics. METHOD: descriptive, exploratory and qualitative study, realized in five polyclinics of a large city in the state of Bahia, Brazil. Semi-structured interviews were conducted with the five nursing supervisors and analyzed using the content analysis technique. RESULTS: the nursing supervisors reported as the main limits for their work the resistance of the team to participate in educational events, a precarious relationship between user demands, insufficiency in the number of nursing staff, difficulty in regulating users, ineffective human and financial resources. Among the possibilities, communication, interpersonal relationships and good coordination between management and coordination were reported. CONCLUSION: the limits found confirm the difficulties encountered in the management of health services, as well as attesting the complexity and need of nursing management to promote good practices for qualified care.

DESCRIPTORS: Nursing. Nursing supervisory. Health centers. Health services administration. Organization and administration 


\section{Introdução}

As concepções sobre a supervisão refletem a organização do processo de trabalho de supervisão em saúde no Brasil e configuram um pensar racional fundamentado nas teorias administrativas, que é fundamentado na racionalidade instrumental ${ }^{1}$.

A supervisão pode ser tradicional e social. O processo de trabalho de supervisão tradicional envolve planejamento, execução e avaliação das atividades a serem realizadas, através da utilização de técnicas e instrumentos que visam aferir eficiência, eficácia e efetividade, proporcionando o desenvolvimento individual, grupal e de relacionamento interpessoal da equipe de enfermagem e qualidade da intervenção prestada aos usuários do sistema de saúde. Por sua vez, o processo de trabalho de supervisão social busca desenvolver relações horizontalizadas entre os profissionais, ao aproximar e sensibilizar a equipe de saúde na resolução das demandas e das necessidades de saúde dos usuários com ações integrais, universais e equânimes ${ }^{1-3}$.

Assim, a supervisão de enfermagem, é uma ferramenta administrativa importante para a condução de uma assistência de qualidade e assume características particulares em relação à forma de exercê-la, os agentes responsáveis pelo seu exercício podem ocupar posições diferentes e atuarem em diferentes níveis hierárquicos. Como instrumento de trabalho, a supervisão de enfermagem se constituiu a partir do surgimento da administração, sendo campo específico de saber e de prática4.

$\mathrm{Na}$ administração dos serviços de enfermagem, a supervisão ou gerência corresponde a uma das principais funções da enfermeira, cabendo a responsabilidade técnica nas instituições de saúde, a planejar atividades de educação, a realizar o dimensionamento do pessoal de enfermagem, além de desempenhar os papeis de avaliação de desempenho e prover a organização de recursos físicos, financeiros e de materiais ${ }^{5}$.

De acordo com a Lei $n^{\circ} 7.498 / 866$, que regulamenta o exercício profissional do pessoal de enfermagem, cabe à enfermeira privativamente as atividades de planejamento, organização, coordenação, execução e avaliação dos serviços de assistência de enfermagem em instituições de saúde, públicas e privadas, e em programas de saúde. Considera-se, portanto, que as atividades de assistência e gestão, sob a forma de cuidado direto às pessoas e supervisão, por exemplo, são importantes pressupostos à prestação de serviços de enfermagem de qualidade ao paciente nos diversos serviços de saúde, como em hospitais, unidades básicas de saúde, unidades de pronto atendimento e em policlínicas.

De forma geral, as policlínicas "visam prestar serviços de média complexidade na rede de saúde da região, ampliando o acesso ambulatorial às especialidades médicas diversas e exames em busca de uma maior atenção à saúde do paciente" ${ }^{\prime \prime}$. Em outras experiências $^{8}$, as policlínicas podem atuar como unidades de pronto atendimento às urgências e emergências, funcionando 24 horas e como porta de entrada para estabilização de pacientes críticos até a sua transferência/ regulação para unidades especializadas, compondo assim uma rede organizada de atenção à saúde? .

Nas unidades atendimento às urgências e emergências, as enfermeiras gestoras precisam lidar continuamente com problemas diários acerca do atendimento nessas unidades, o grande fluxo de pacientes atendidos $^{9}$, a falta de recursos humanos, físicos e materiais ${ }^{5}$, além de problemas de ordem organizacional da rede de atenção à saúde ${ }^{4}$. Por isso, as enfermeiras que atuam como supervisoras desses serviços precisam desenvolver competências diversas como liderança, de iniciativa, de maturidade e equilíbrio emocional.

Diante destas considerações, é justificada a escolha deste tema para estudo por reconhecer na supervisão de enfermagem uma importante tecnologia para o trabalho da enfermeira, em particular para aquelas que ocupam o cargo de supervisoras de enfermagem nas policlínicas.

Tomando como referências estas reflexões, este estudo pretende responder à seguinte questão: quais são os limites e possibilidades do processo de trabalho de supervisão relatados por enfermeiras das policlínicas? 
Desta maneira, este estudo tem por objetivo descrever os limites e as possibilidades do processo de trabalho de supervisão de enfermagem em policlínicas.

\section{Método}

Trata-se de estudo descritivo, exploratório e de abordagem qualitativa.

O estudo foi realizado nas policlínicas, de uma grande cidade do interior do Estado da Bahia. As seis policlínicas da cidade estão localizadas na zona urbana, são administradas pela gestão municipal, realizam atendimento ambulatorial em diversas especialidades médicas, são ordenadoras dos fluxos de urgência e algumas funcionam também 24 horas como pronto atendimento às emergências.

A população foi composta pelas enfermeiras supervisoras das seis policlínicas que atenderam aos critérios de inclusão: ocupar o cargo há pelo menos seis meses (tempo considerado pelas pesquisadoras como necessários para conhecer os processos gerenciais) e estar em pleno exercício do cargo. Foi excluída uma supervisora, por estar de licença no momento da coleta dos dados. Assim, os participantes desse estudo foram cinco supervisoras de enfermagem.

A coleta dos dados foi realizada por meio de uma entrevista semiestruturada durante o período de maio 2015 e junho 2015 e tendo como questões norteadoras: "quais são os limites que você vivencia no processo de supervisão em enfermagem? Quais são as possibilidades encontrados no processo de trabalho de supervisão em enfermagem que você desenvolve?" As entrevistas foram realizadas em sala privada, em horário previamente agendado, gravada em formato MP3 e posteriormente arquivada em formato CD-ROM. O tempo médio de cada entrevista foi de 15 minutos.
A análise dos dados foi realizada pela técnica de análise de conteúdo, conforme os estudos de Minayo ${ }^{10}$. Foi sistematizada em três etapas: ordenação, classificação e análise final dos dados. A ordenação dos dados envolveu a transcrição das entrevistas gravadas e a leitura do material empírico. A classificação dos dados foi a fase mais longa. Foi feita uma leitura exaustiva do material empírico tendo como base o referencial teórico e os pressupostos do estudo. Foram levantados os temas mais incidentes e relevantes com delineamento das unidades temáticas: resistência da equipe de enfermagem em participar de eventos educativos e resistência a mudanças; educação permanente em saúde. Com as unidades temáticas descritas foi possível criar duas categorias analíticas: limites do processo de trabalho e possibilidades para o processo de trabalho de supervisão em policlínicas na percepção da enfermeira supervisora. A análise final dos dados deu-se com a interpretação dos resultados obtidos por meio da articulação do material empírico com o teórico.

Assegurou-se o anonimato dos participantes e das unidades de saúde pesquisadas, por isso os participantes foram nomeados conforme elementos do sistema solar, sendo eles: Sol, Lua, Estrela, Cometa e Vênus, e as unidades de saúde de A a E. Além disso, todos os participantes concordaram em participar da pesquisa assinando o Termo de Consentimento Livre e Esclarecido.

Os aspectos éticos foram preservados nesta pesquisa e em acordo com a resolução $n^{\circ} 466 / 2012$ do Conselho Nacional de Saúde, obtendo, portanto, aprovação do Comitê de Ética e Pesquisa da Universidade Estadual de Feira de Santana com a pesquisa registrada sobre o parecer $n^{\circ} 1.084 .055$ (CAAE 42651715.8.0000.0053). A fim de garantir o rigor na pesquisa qualitativa, adotou-se o check list da pesquisa qualitativa presente nas diretrizes do Consolidated Criteria for Reporting Qualitative Research (COREQ). 


\section{Resultados}

As cinco supervisoras entrevistadas possuíam graduação em enfermagem, idade entre 25 e 45 anos, sendo três do sexo feminino e dois masculinos. 0 tempo médio de exercício profissional como supervisora da policlínica foi entre 6 meses e 3 anos.

\section{Limites do processo de trabalho de supervisão em policlínicas}

Com relação as situações geradoras de limites destacam-se: resistência da equipe de enfermagem em participar de eventos educativos, resistência para mudança, relação precária entre demandas de usuários e quantitativo da equipe de enfermagem, dificuldade na regulação de usuários e recursos humanos e financeiros ineficazes. Os fragmentos a seguir destacam esses fatores.

[...] então isso acaba problematizando nosso processo de supervisão, [...] sensibilizar essa pessoa acaba sendo difícil, que tem 30 anos de prática [...] que acaba não indo para cursos, quando se espera por mudança [...].

[...] os maus hábitos que algumas técnicas têm, de longa data, não é? As técnicas mais antigas na casa, a gente enfrenta algumas dificuldades em relação a isso; a gente tem de corrigir e as vezes a gente não consegue.

(Estrela)

[...] trabalhar em cima dessas falhas através da educação continuada e permanente dos funcionários [...] alguns treinamentos, alguns cursos, atualização durante todo o ano, além das que a gente faz interna durante todo o ano [...]. (Vênus)

A relação precária entre demanda de usuários e quantitativo da equipe de enfermagem foi outro aspecto sinalizado pelos participantes do estudo como limites para o desenvolvimento do processo de trabalho de supervisão realizado pela enfermeira nas policlínicas em nível local. É o que se observa nas falas seguintes:

[...] quem decide a porta de entrada do paciente é o próprio paciente. [...] existem muitas demandas, as vezes de pacientes que tem uma necessidade ambulatorial, o problema dele é de atenção básica

[...] porque é um serviço 24 horas e que tem uma demanda muito grande. Então eu acho que é uma das coordenações mais difíceis que a gente tem hoje [...].
Existem limites quanto a gestão de pessoas, que é um número até então limitado de profissionais, onde a gente trabalha com uma demanda de profissionais abaixo da nossa necessidade, que aumentou muito o quantitativo de atendimento, ou seja, eu precisaria mais de profissionais, de enfermeiros [...]. (Cometa)

Outro fator limitante para o desenvolvimento do processo de trabalho de supervisão nas policlínicas em nível local no olhar da enfermeira supervisora é a dificuldade na regulação de usuários, como é descrito nos fragmentos a seguir:

\section{[...] outra dificuldade é a questão também do suporte} que o pronto atendimento tem de ter em relação a unidades hospitalares, hoje a nossa referência é o Cleriston Andrade, o fato de que a gente precisa transferir esse paciente que necessita de uma atenção hospitalar e a gente às vezes não consegue essa vaga com tanta facilidade. (Lua)

[...] em questão de regulação de paciente, que a gente tem uma certa dificuldade, por conta do único hospital de referência ser o Cleriston [...]. (Cometa)

\section{Possibilidades para o processo de trabalho de supervisão em policlínicas}

A comunicação, o bom relacionamento interpessoal, a interação com os membros da equipe de enfermagem o apoio da coordenação central da Secretaria Municipal de Saúde, da coordenação geral das policlínicas e a satisfação no trabalho desenvolvido foram destacadas como possibilidades para o bom desenvolvimento da supervisão, conforme relato das entrevistadas:

[...] a gente tem um bom relacionamento, o que facilita bastante [...]. (Estrela)

[...] a maior facilidade que eu tenho hoje é a equipe com que eu trabalho [...] se você não tiver uma equipe boa, e qualificada, no sentido de ta ali disposta realmente a resolver os problemas, cheguei pra trabalhar e vou tentar sanar os problemas dentro das minhas possibilidades, assim, acho que esse apoio é muito grande, dentro da própria equipe [...]. (Lua)

A facilidade é a comunicação, não é? A comunicação facilita muito, onde eu trabalho com observação direta em relação à supervisão [...] isso a gente mantém a comunicação com os profissionais, com isso a gente consegue atingir alguns objetivos. (Cometa) 
O apoio da coordenação central da Secretaria Municipal de Saúde, da coordenação geral das policlínicas e a satisfação no trabalho desenvolvido foram pontuados pelas enfermeiras supervisoras como facilidades para o desenvolvimento do processo trabalho de supervisão, como é destacado nos fragmentos a seguir:

[...] Graças a Deus nós temos a facilidade de junto a Secretaria, não é? Nós temos uma supervisão efetiva, e uma coordenação, também bastante efetiva junto à nossa unidade, não é? E isso facilita nosso processo todo [...] A coordenação geral também, ela reconhece nosso trabalho; [...] e é uma boa conduta para que a gente continue nessa forma, dessa forma. (Estrela)

[...] a supervisão enquanto coordenação geral das policlínicas. [...] se eu não tivesse enfermeiras para também estar ajudando nesse sentido seria complicado entendeu. (Lua)

[...] a gente tem o apoio da coordenação, para nos ajudar também, essa parte. E a Secretaria também tem nos ajudado, a Secretaria Municipal de Saúde. [...] tem as supervisoras, cada policlínica tem uma supervisora, uma coordenadora geral [...]. (Vênus)

[...] eu faço com amor, eu amo o que eu faço, [...] mesmo com todas suas dificuldades [...]. (Cometa)

\section{Discussão}

A enfermagem, em seu processo de trabalho, tem por funções, cuidar, assistir, gerenciar, administrar, pesquisar, investigar, ensinar e orientar. Assim, diante dessas atividades o enfermeiro tem o papel de supervisionar o pessoal de enfermagem nas unidades de saúde, contribuindo para o desempenho do trabalho e orientando a equipe na concretização dos serviços ${ }^{11}$.

Neste estudo a gestão dos serviços apresentou limites na operacionalização do processo de trabalho de supervisão, assim como possibilidades. Acredita-se que para o processo de trabalho de supervisão de enfermagem nas policlínicas seja desenvolvido de maneira eficaz, a supervisão de enfermagem deve conhecer as ferramentas que norteiam a sua operacionalização, possibilitando assim a identificação e superação desses limites.
A partir dos resultados do estudo, é possível perceber que há resistência da equipe de enfermagem em participar de eventos educativos. A educação em serviço, continuada ou permanente na área da saúde $^{12}$ é de grande relevância para o aprimoramento e capacitação dos profissionais, em todos os níveis da atenção, seja na atenção básica ou em urgências e emergências.

Entretanto, o estudo de Bugs et al. ${ }^{12}$ revelou que, apesar das tentativas de capacitação pelos supervisores das unidades de saúde, há ainda forte resistência dos profissionais atuantes em participar das atividades de educação em serviço. Com base nessa contestação, é possível observar que há convergência entre o referido estudo e o resultado desta pesquisa, em que aparece claramente a dificuldade das enfermeiras líderes no desenvolvimento do processo de trabalho de supervisão em motivar as técnicas de enfermagem a participarem de ações educativas.

Neste sentido, cabe às supervisoras de enfermagem orientar, supervisionar e propor atividades que possam integrar toda a equipe de saúde. É necessário desenvolver em cada profissional de saúde, a sensibilização da importância de sua participação para a melhor continuidade do desenvolvimento do processo de trabalho de supervisão e, consequentemente, o melhoramento da assistência prestada aos usuários.

Para o melhoramento da assistência aos usuários, é preciso que esses também sejam informados, capacitados e conscientizados acerca dos serviços que são prestados pela unidade de saúde as quais procuram atendimento, pois muitos casos atendidos em unidades de pronto atendimento, frequentemente, não se enquadram como urgência ${ }^{13}$, gerando assim um fluxo maior de pessoas, excede a capacidade das unidades e superlota as unidades ${ }^{14}$. Portanto, cabe à enfermeira supervisora utilizar as técnicas e os instrumentos disponíveis aliados aos seus conhecimentos sobre o processo de trabalho de supervisão, para gerenciar a tomada de decisão do problema em questão.

Outro fator limitante para o desenvolvimento do processo de trabalho de supervisão de enfermagem nas policlínicas é em relação aos entraves na regulação de usuários para unidades e hospitais especializados. 
Esta situação faz com que as enfermeiras supervisoras se envolvam, priorizando as articulações de transferências de pacientes e deixem de forma secundária a supervisão. Assim, conforme aponta Martins e Alves ${ }^{15}$ a gestão de leitos, transferência e regulação de pacientes é um nó crítico que os coordenadores e gestores das unidades de emergência têm enfrentado continuamente dentro da rede de atenção à saúde.

Por outro lado, estudos têm apontado um elenco de fatores limitantes no exercício do processo de trabaIho de supervisão que convergem para os resultados deste estudo, tais como: deficiência de recursos materiais e humanos quanti-qualitativos, ambiente físico das unidades inadequado, o sistema de contra referência de saúde ${ }^{1-3}$. Por outro lado, esses mesmos estudos sinalizam para outros fatores limitantes que não aparecem neste estudo e que se aliam ao ensino, ao controle e ao poder como aspectos negativos preponderantes, que são: falta de autonomia profissional, interferência política partidária e ingerência, baixa remuneração, relações interpessoais difíceis, insatisfação dos profissionais e da equipe com o trabalho, a sobrecarga de atividades de assistência, relações assimétricas de poder, a falta de resolubilidade ocasiona sentimentos de culpa e impotência, o exercício de práticas reiterativas na saúde, acesso ao transporte e aos domicílios, a violência, a falta de tempo e o modo de fazer centrado em procedimentos técnicos, caracterizando assim um processo de trabalho de supervisão tradicional1-3.

Entendemos que os fatores que limitam e interferem no processo de trabalho de supervisão de enfermagem em policlínicas são determinados pelo contexto histórico, social e político da sociedade, ou seja, estão inseridos nos componentes políticos, na estrutura organizacional do serviço, na formação dos profissionais de saúde e na gestão dos serviços de saúde, evidenciando, por parte destes, a falta de percepção da supervisão enquanto processo social.

Estes determinantes que limitam e interferem no processo de trabalho de supervisão se constituem em espaços de atravessamento, que tendem à manutenção do que está, mas, podem se constituir em espaços de transversalidade, favoráveis às mudanças. E para isso, faz-se mister o entendimento da supervisão como estratégia/ferramenta/tecnologia gerencial, é preciso repensar as relações interpessoais, o cuidado, a ética no agir e ser no processo decisório.
A supervisão não pode ser entendida de modo desarticulado do quadro institucional e social mais geral, é necessário avançar na compreensão do processo de trabalho de supervisão social que contemple as questões subjetivas, sociais e estratégicas, portanto necessariamente psicossociais, políticas e ideológicas extrapolando a lógica da gestão e da organização

Nesta perspectiva, a comunicação e o relacionamento interpessoal têm se destacado como possibilidades para o enfrentamento dos desafios encontrados durante o desenvolvimento do processo de trabalho de supervisão e o gerenciamento das enfermeiras nas policlínicas. A comunicação é uma ferramenta utilizada no desenvolvimento para o planejamento do processo de trabalho da supervisão de enfermagem ${ }^{16-17}$, constituindo-se em estratégia que possibilita mudanças requeridas no modo compartilhado para o bom relacionamento interpessoal entre 0 grupo de trabalho.

Assim, a supervisão de enfermagem se configura como ferramenta para o enfrentamento dos limites encontrados nesta pesquisa. A sua implementação nos serviços de saúde auxiliará na condução do processo de trabalho, por meio do desenvolvimento de conhecimento, habilidades e no suporte para o pessoal supervisionado ${ }^{18}$.

As limitações deste trabalho dizem respeito a escolha da técnica de coleta dos dados e a técnica da análise dos dados qualitativos, pois ambas podem sofrer influências diversas no momento da coleta dos dados, como a questão da neutralidade do pesquisador. Por isso, novos estudos são sugeridos na mesma temática e sob a abordagem de métodos mistos.

\section{Conclusão}

Com este estudo foi possível aproximar o conhecimento acerca dos limites e possibilidades do processo de trabalho de supervisão em enfermagem em policlínicas. Constatou-se que os principais limites para a supervisão em unidades de policlínicas com os quais as enfermeiras defrontam-se são em relação à resistência da equipe de enfermagem em participar de eventos educativos, resistência para mudança, relação precária entre demandas de usuários, insuficiência no quantitativo da equipe de enfermagem, dificuldade 
na regulação de usuários e recursos humanos e financeiros ineficazes. Entre as possibilidades para a superação desses limites destacaram principalmente a comunicação, o relacionamento interpessoal e a boa articulação entre a gestão e coordenação.

Ademais, os resultados corroboram para a justificativa de que o processo de supervisão não se concentra apenas na pessoa instituída do papel de supervisor, mas em toda a equipe para o bom andamento, condução e resolutividade das ações.

\section{Contribuições dos autores}

Sacramento LA colaborou na concepção, delineamento da pesquisa, coleta dos dados, redação, análise, revisão crítica do artigo e aprovação final da versão a ser publicada. Figueredo WN , Servo MLS, Macêdo TTS e Fernandes ETBS colaboraram no delineamento da pesquisa, na redação, análise, interpretação dos dados, revisão crítica do artigo e aprovação final da versão a ser publicada.

\section{Conflitos de interesses}

Nenhum conflito financeiro, legal ou político envolvendo terceiros (governo, empresas e fundações privadas, etc.) foi declarado para nenhum aspecto do trabalho submetido (incluindo, mas não se limitando a subvenções e financiamentos, participação em conselho consultivo, desenho de estudo, preparação de manuscrito, análise estatística, etc.).

\section{Referências}

1. Santana TS, Servo MLS, Borges RBV. Representações Sociais do enfermeiro sobre o estresse no processo de trabalho de supervisão hospitalar em unidade de emergência. In: MissiasMoreira R, Freitas VLC, Collares-da-Rocha JCC, organizadores. Representações Sociais na contemporaneidade - volume 1. Curitiba: CRV; 2019.

2. Servo MLS, Góis RMO. Representações sociais (re) veladas por enfermeiras da rede de atenção à saúde sobre a supervisão em enfermagem. In: Missias-Moreira R, Sales ZN, Freitas VLC, Oliveira DC, organizadores. Representações sociais, educação e saúde: um enfoque multidisciplinar - volume 3. Curitiba: CRV; 2017.

3. Dias CA, Santos DC, Matias LO, Servo MLS, Santana CLA, Tanaka LH. Nursing supervision from the perspective of nurse coordinators of a teaching hospital. Rev Baiana Enferm. 2018;32(e27422):1-12. doi: 10.18471/rbe.v32.27422
4. Chaves LDP, Mininel VA, Silva JAM, Alves LR, Silva MF, Camelo SHH. Supervisão de enfermagem para integralidade do cuidado. Rev Bras Enferm. 2017;70(5):1165-70. doi: 10.1590/0034-71672016-0491

5. Ferreira VHS, Teixeira VM, Giacomini MA, Alves LR, Gleriano JS, Chaves LDP. Contribuições e desafios do gerenciamento de enfermagem hospitalar: evidências científicas. Rev Gaúcha Enferm. 2019;40(e20180291):e20180291. doi: 10.1590/1983$\underline{1447.2019 .20180291}$

6. Decreto n. 94.406/87, de 30 de março de 1987. Regulamenta a Lei no 7.498 , de 25 de junho de 1986. Dispõe sobre o exercício da Enfermagem, e dá outras providências [Internet]. Dário Oficial da Republica Federativa do Brasil. 1986 mar. 30. Disponível em: http://www.planalto.gov.br/ccivil_03/decreto/1980-1989/D94406. $\mathrm{htm}$

7. Secretaria da Saúde do Estado da Bahaia -SESAB. Policlínicas regionais de saúde [Internet]. 2020. Disponível em: http://www.saude.ba.gov.br/municipios-e-regionalizacao/ policlinicasregionais/

8. Prefeitura Municipal de Feira de Santana. Secretaria Municial de Saúde. Urgências e Emergências - Políclínicas [Internet]. 2020. Disponível em: http://www.feiradesantana.ba.gov.br/servicos. asp?id=14\&link=sms/urgenciaemergencia/policlinica.asp

9. Konder MT, O'Dwyer G. As unidades de pronto-atendimento na Política Nacional de Atenção às Urgências. Physis. 2015;25(2):52545. doi: 10.1590/S0103-73312015000200011

10. Minayo MCS. O desafio do conhecimento: pesquisa qualitativa em saúde. São Paulo: Hucitec; 2013.

11. Carvalho NA, Gama BMBM, Salimena AMO. A supervisão sob a ótica dos enfermeiros: reflexos na assistência e trabalho em equipe. Rev Adm Saúde. 2017;17(69):1-18. doi: 10.23973/ras.69.68

12. Bugs TV, Rigo DFH, Bohrer CD, Borges F, Oliveira JLC, Tonini NS. Dificuldades do enfermeiro no gerenciamento da unidade de pronto-socorro hospitalar. Rev Enferm UFSM. 2017;7(1):90-9. doi: $\underline{10.5902 / 2179769223374}$

13. Oliveira SN, Ramos BJ, Piazza M, Prado ML, Reibnitz KS, Souza AC. Emergency Care Units (UPA) 24h: the nurses' perception. Texto Context - Enferm. 2015;24(1):238-44. doi: 10.1590/0104$\underline{07072015003390011}$

14. Roncalli AA, Oliveira DN, Silva ICM, Brito RF, Viegas SMF. Protocolo de Manchester e população usuária na classificação de risco: visão do enfermeiro. Rev Baiana Enferm. 2017;31(2):e16949. doi: 10.18471/rbe.v31i2.16949

15. Martins BR, Alves M. O processo de trabalho do enfermeiro na unidade de urgência e emergência de um hospital público. Rev Med Minas Gerais. 2018;28(Supl 5):e-S280519. 
16. Leite TMC, Vergílio MSTG, Silva EM. Pediatric nurse's work process: a reality to be transformed. Rev Rene. 2017;18(1):26-34. doi: $10.15253 / 2175-6783.2017000100005$

17. Figueredo WN, Véras RM. Integrando Educação e Trabalho: o caso do Permanecer SUS da Secretaria da Saúde do Estado da Bahia. Trab Educ Saúde. 2016;14(3):803-23. doi: 10.1590/19817746-sol00017
18. Pinto DJE, Santos MR, Pires RM. Relevance of indicators of clinical supervision strategies in nursing. Rev Rene. 2017;18(1):1925. doi: $10.15253 / 2175-6783.2017000100004$ 